\title{
LOCALIZED BIONANORECEPTOR 3D-ASSEMBLY VIA ELECTROWETTING: AN INTEGRATED MICRO/NANO/BIO FABRICATION TECHNOLOGY
}

\author{
S. Chu ${ }^{l}$, F. Zang ${ }^{l}$, A.D. Brown ${ }^{2}$, J.N. Culver ${ }^{2}$, and R. Ghodssi $i^{*}$
}

${ }^{1}$ Department of Electrical and Computer Engineering, Institute for Systems Research, ${ }^{2}$ Institute for Bioscience and

Biotechnology Research and Department of Plant Science and Landscape Architecture

University of Maryland, College Park, Maryland, USA

\section{ABSTRACT}

This work presents localized functionalization of bionanoreceptors, genetically modified Tobacco mosaic viruses, on high-aspect-ratio micropillar arrays using electrowetting principles. This approach allows wetting of the structurally hydrophobic component with TMV solution resulting in a near-complete coverage ( $\sim 95 \%)$ of TMVs over the dense microstructures with clear functionalization boundaries. The functionalization morphology and biochemical activity of the electrowetted TMVs are confirmed using SEM and fluorescent labeling, respectively. A 5-fold increase in fluorescence intensity is achieved with $\mu$ PAs over planar substrate. The TMV-based hierarchical functional structure enabled by electrowetting can serve as a high-surface-area component for next generation micro-devices.

\section{INTRODUCTION}

Recently, integration of biological molecules into microfabrication processes has been extensively explored for the development of a wide-range of micro-/nano-devices. Their natureinherited structural and functional versatility combined with advances in biology and bioengineering has great advantages for creating tailored nanoscale functionalities on devices for a wide range of applications including energy storage and sensing devices [1].

Tobacco mosaic virus (TMV) is one of the most extensively studied biological macromolecules which has been successfully integrated with various microfabrication processes. It is a plant virus featuring a high-aspect-ratio (HAR) structure $(300 \mathrm{~nm}$ in length and $18 \mathrm{~nm}$ in diameter) with an ultra-high density of identical functional groups expressed on their surfaces [1]. Previously, the advancement of genetic/chemical engineering technologies allowed modification of the TMVs to be used as either biological or chemical sensing probes on microfabricated transducers [2-5]. Also, cysteine-modification of wild-type TMVs (TMV-1cys) enabled integration of the nanoscaffolds into microfabrication processes via self-assembly and electroless metallization to create high-surfacearea energy storage devices [6]. Hierarchical electrodes, where nanoscale TMVs are combined with microscale structures, amplified electrode surface areas even more so [7]. The high surface area functional architecture is very attractive not only for energy storage devices but also for on-demand miniaturized sensing platforms where would benefit from the very dense bio/chemical recognition events for higher sensitivities. However, access of TMV solution into HAR microcavities has been limited and hindered further increase of device surface area, a phenomenon attributed to the hydrophobic nature of the dense $\mu$ PAs [8].

Here, we address the aforementioned challenges through the use of electrowetting principles for localized functionalization of biological receptors (TMV-1cys) from solution on high surface area microstructure. Electric potential applied across the interface between TMV droplets and HAR- $\mu$ PAs results in a breakdown of structural hydrophobicity, driving down the TMV solution into the vertical microcavities. The access of TMV solution in the lateral direction is self-limited due to the surface tension created at the wetting boundaries allowing localized bionanoreceptor functionalization. The resulting high-surface-area structures with the electrically or chemically functional surfaces bring great advantages for the development of TMV-based energy storage devices or bio/chemical sensing platforms.

\section{MATERIALS AND METHODS}

Structural hydrophobicity of Au-coated Si- $\mu$ PAs
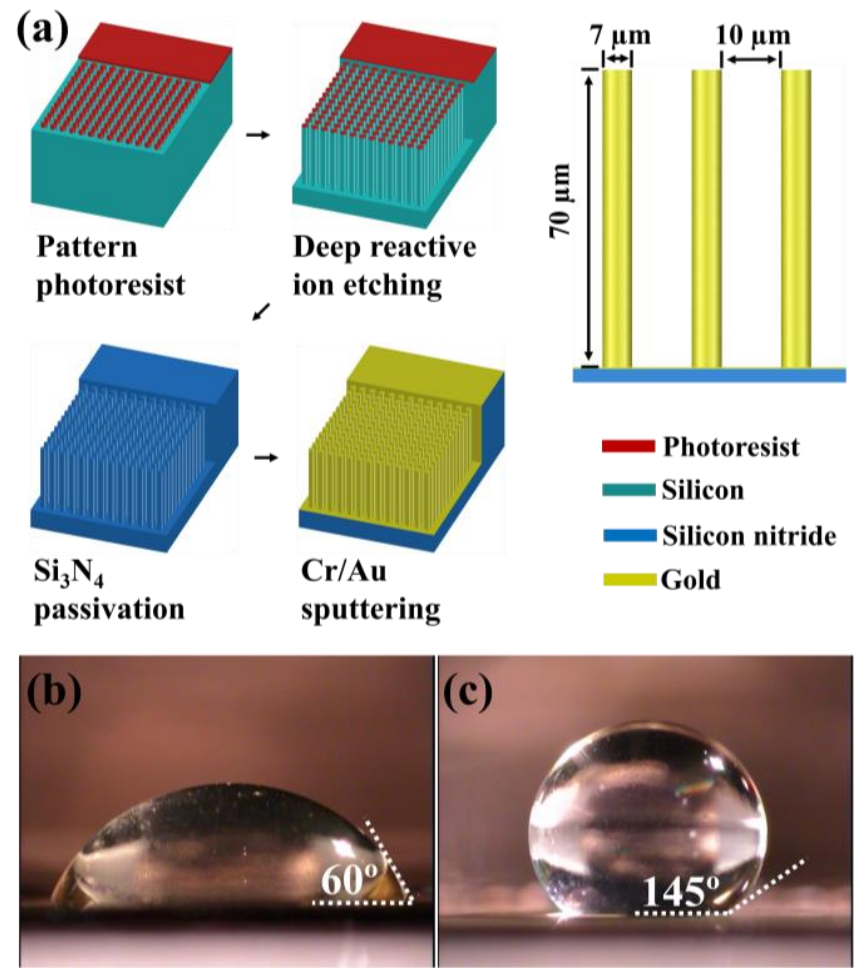

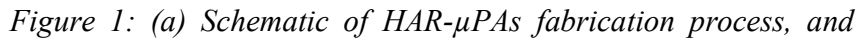
optical microscopy of $10 \mu \mathrm{l} T M V$ droplet on (b) planar Au and (c) HAR- $\mu$ PAs surfaces.

The fabrication process for the HAR- $\mu$ PAs is described in Fig. 1a. A Si wafer is etched down via DRIE using a patterned negative photoresist (NR9-1500PY, Futurrex) as the etch mask. The resulting Si micropillar arrays are passivated with $300 \mathrm{~nm}$ PECVD $\mathrm{Si}_{3} \mathrm{~N}_{4}$ followed by sputtering of $\mathrm{Cr}(30 \mathrm{~nm}) / \mathrm{Au}(150 \mathrm{~nm})$. The footprint of the final $\mu \mathrm{PA}$ electrode is $1 \mathrm{x} 1 \mathrm{~cm}^{2}$ with $10: 1(70 \mu \mathrm{m}$ in height, $7 \mu \mathrm{m}$ in diameter) and 7:1 (70 $\mu \mathrm{m}$ in height, $10 \mu \mathrm{m}$ spacing between nearest pillars) aspect ratios for pillars and spacings, respectively. Such geometry of the $\mu$ PAs is selected for this work based on our previous studies which showed limited TMV assembly on $\mu \mathrm{PA}$ densities higher than the selected geometry [8]. Also, the HAR- $\mu$ PAs with spacing aspect ratio higher than 7:1 showed unstable wettability transition during the electrowetting step (data not shown). This is expected to be due to a poor coating of sputtered $\mathrm{Cr} / \mathrm{Au}$ inside the deep micro cavities limiting electric potential to be 
applied at the droplet- $\mu$ PAs interfaces. Considering the importance of high spacing aspect ratio geometry for increasing both the surface area and bionanoreceptor densities, a method to make good electrical connections between the dense micro-network is critical for the success of this technology.

Fig. $1 \mathrm{~b}$ and $1 \mathrm{c}$ demonstrates the wettability of HAR- $\mu$ PAs by comparing the contact angle measurement of $10 \mu \mathrm{TMV}$ solution droplet $(0.2 \mathrm{mg} / \mathrm{ml}$ in phosphate buffer $(\mathrm{pH} 7))$ on planar Au (Fig. 1b) and the HAR- $\mu$ PAs (Fig. 1c) surfaces. While the planar Au surface exhibits hydrophilic nature with $60^{\circ}$ contact angle, the HAR- $\mu$ PAs results in hydrophobic characteristic measuring $145^{\circ}$ contact angle. This is close to the theoretically expected value calculated based on the Cassie equation (1), where $\theta^{*}$ is the apparent contact angle on the HAR- $\mu$ PAs, $\phi_{\mathrm{s}}$ is the fraction of the solid in contact with the TMV solution, and $\theta_{0}$ is the contact angle on the planar $\mathrm{Au}$ substrates [9-10]. The TMV droplet on HAR- $\mu$ PAs are readily movable with no traceable liquids remaining on the electrode surface.

$$
\cos \theta^{*}=-1+\phi_{S}\left(1+\cos \theta_{0}\right)
$$
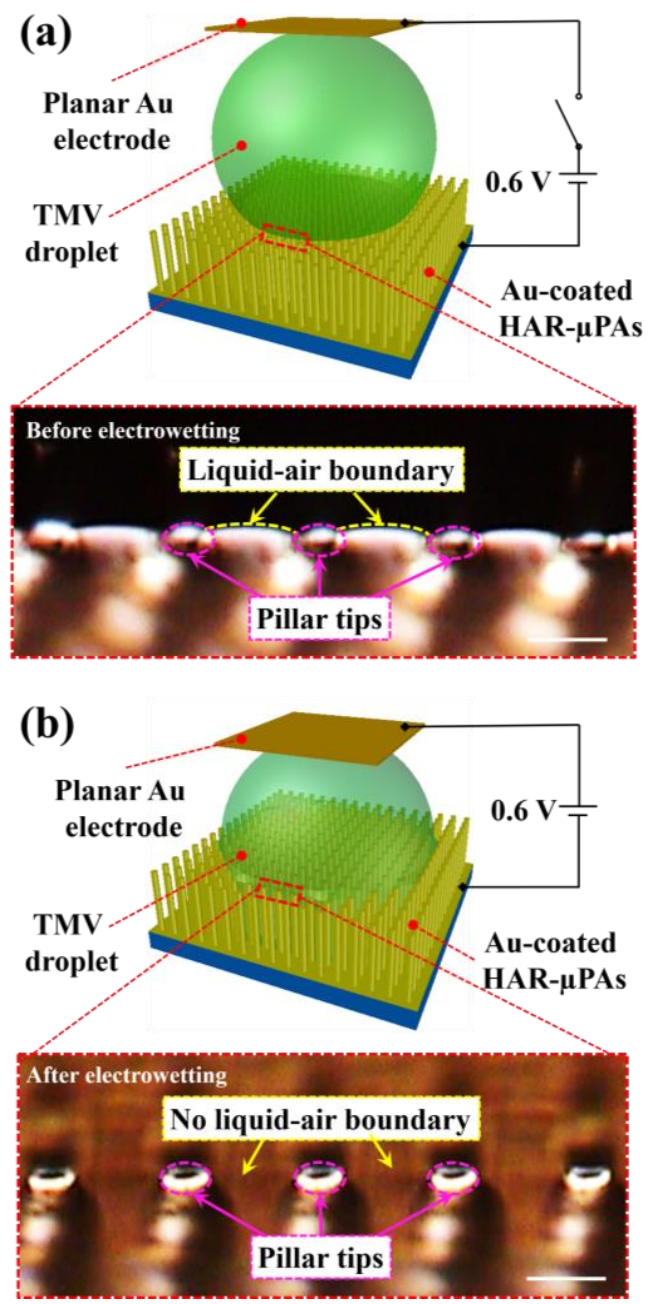

Figure 2: Schematics of the TMV droplet on $\mu$ PAs (top) with optical microscopy from the top of the $\mu$ PAs interfacing droplet edges (bottom). The (a) Casie-Baxter state and (b) the Wenzel state of the droplet is clearly observed in the microscopy images (focusing at edge-on pillar tips) taken before and after electrowetting, respectively (scale bars: $10 \mu \mathrm{m}$ ).

\section{Structural wettability transition via electrowetting}

The initial wetting state of the TMV droplet on HAR- $\mu$ PAs can be described as Casie-Baxter state where liquid-air boundaries form on the very top of the $\mu$ PAs as shown in Fig. 2a. The top end of each micropillars function as an arrayed surface defect where the pinning of the liquid phase limits the access of the solution into the vertical cavities. It is well-known that an electric potential can induce transition in structural wettability (from Casie-Baxter to Wenzel state) by reducing the surface tension at the solid-liquid boundaries [10]. As described in Fig. 2b, an electric potential of 0.6 $\mathrm{V}$ (DC) is applied for 1 minute by sandwiching a $10 \mu 1$ droplet between the bottom $\mu$ PAs and a planar Au electrode placed on top of the droplet using a 3D-printed $1 \mathrm{~mm}$-thick spacer. The electrowetted HAR- $\mu$ PAs are incubated overnight (18 hours) at room temperature for the self-assembly of TMV-1cys onto Au surfaces. The electrodes were placed in a plastic boxes sealed with parafilm to prohibit sample drying during the self-assembly.

After the overnight self-assembly step the electrodes are rinsed in phosphate buffer solution in $\mathrm{pH} 7$ (PB), followed by either a standard metallization process, which involves palladium surface activation and electroless nickel coating [6], or a fluorescent labeling step to be evaluated in SEM and fluorescent microscopy, respectively. For the fluorescence analysis, each electrode is rinsed in PB after the overnight self-assembly process, and a drop of $50 \mu \mathrm{l}$ Fluoresceine-5-Maleimide reagent (excitation: $494 \mathrm{~nm}$, emission: $518 \mathrm{~nm}$, Thermo Scientific) solution $(0.3 \mathrm{mg} / \mathrm{ml}$ in PB) is added to each of the electrodes for 2-hour labeling reaction at room temperature. After the labeling reaction, the electrodes are rinsed in $\mathrm{PB}$, and further immersed in $3 \mathrm{ml} \mathrm{PB}$ for 30 minutes to get rid of any excess/unbound labeling reagents before imaging. A fluorescent microscopy set up (MVX10, Olympus), equipped with a broadband excitation light source and a designated imaging software (cellSens), is used to collect the data. All images are collected for 2 seconds exposure, and the images are analyzed using Image J to compare the differences between the samples.

\section{RESULTS AND DISCUSSIONS}

\section{Morphology of electrowetted TMVs on HAR- $\mu$ PAs}

The side-view optical micrographs shown at the bottom of Fig. $2 \mathrm{a}$ and $2 \mathrm{~b}$ confirm the wettability transition from Casie-Baxter to Wenzel state; while the clear liquid-air boundaries between the liquid-solid pinning points are observed before the electrowetting, no liquid-air boundary is observed on top of the $\mu$ PAs after the electrowetting step. We expected the successful wettability transition by also observing apparently diminished contact angle after the electrowetting step. Droplets sandwiched without electrowetting results in removal from the HAR- $\mu$ PAs surface by the hydrophilic top electrode (planar $\mathrm{Au}$ ) while disassembling the experimental set up.

Comparisons of the cross-sectional SEM images shown in Fig. 3 confirm the successful functionalization of TMVs along the deep microcavities of the HAR- $\mu$ PAs enabled by the electrowetting process. Fig. 3a shows the cross-sectional SEM of TMV-coated micropillars located at the center of the wetted area. Compared to our previous studies, which showed limited TMV coating at the bottom of the pillars (the electrodes were immersed in $1 \mathrm{ml} \mathrm{TMV}$ solution for the self-assembly step) [8], near-complete ( $95 \%)$ TMV coverage of the pillar side-walls is achieved confirming the excellent wetting of the dense microstructure with the TMV solution. Fig. 3 b shows pillars that are exposed to a TMV droplet without electrowetting. Only isolated TMVs are observed with most of the micropillar surfaces exposed with DRIE traces from its twostep etch process. This indicates that the Casie-Baxter state of the droplet is stable throughout the overnight self-assembly process, and 
the present TMVs are likely due to the rinsing or the post metallization processes which could introduce unbound TMV particles into the cavities.

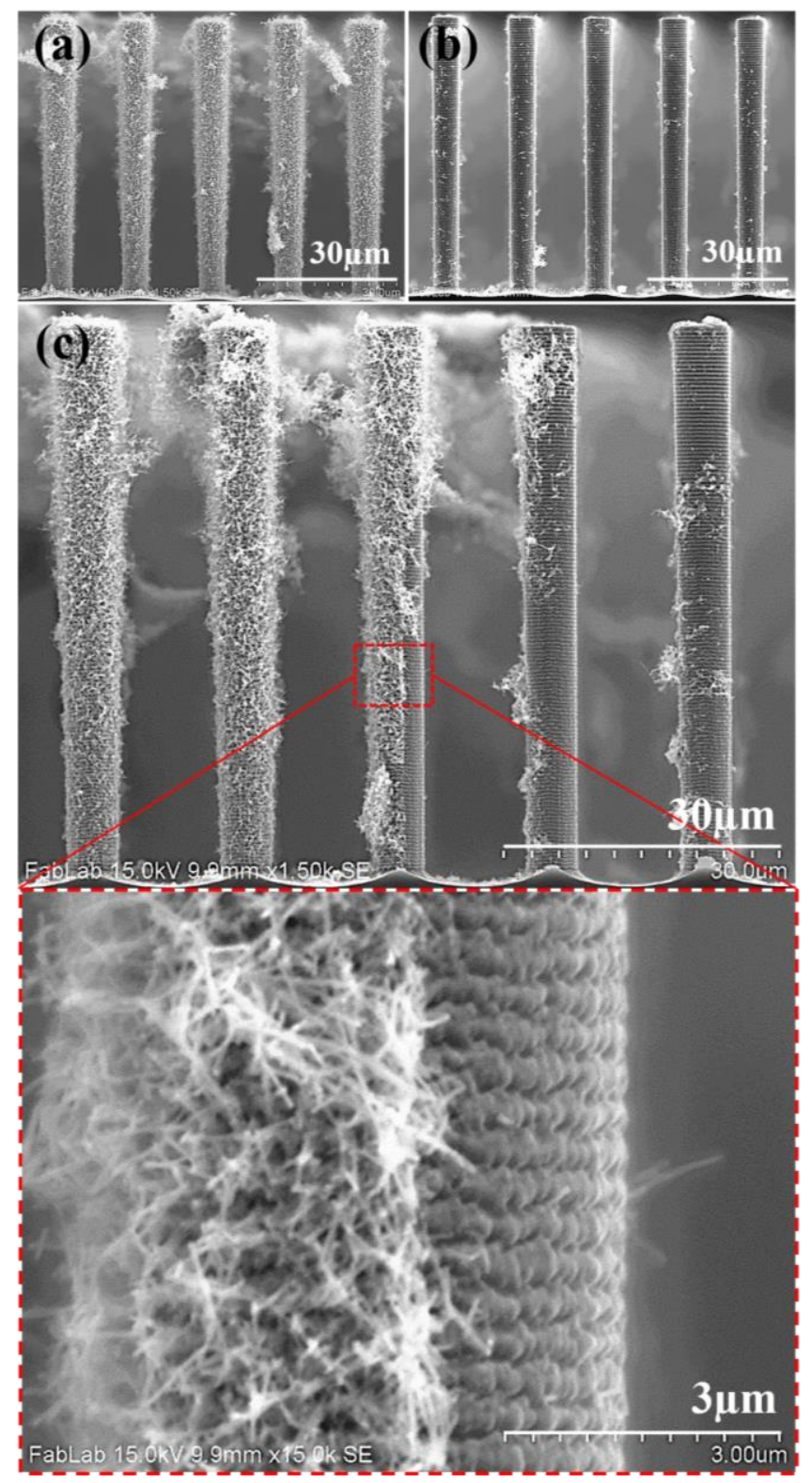

Figure 3. Cross-sectional SEM images of the nanostructured TMVS on the $\mu$ PAs. (a) Electrowetting allowed near-complete TMV coverage along the deep $\mu P A s$ sidewalls compared to the (b) nonelectrowetted $\mu$ PAs. (c) The clear functionalization boundary is observed at the wetting edge demonstrating the discrete nature of the method.

Fig. $3 \mathrm{c}$ shows the electrowetted-TMVs at the wetting edge on HAR- $\mu$ PAs. A distinct boundary is achieved, with a representative single pillar side-wall exhibiting both TMV nanotextures and exposed DRIE etch steps on the wetted and un-wetted sides, respectively. This is attributed to limited lateral access of the TMV solution controlled by surface tension. The initial limitation of the structural hydrophobicity here plays an advantageous role to locally confine the bionanoreceptor functionalization on high surface area microstructures. The localization capability of the electrowetting offer great opportunities for developing TMV-based arrayed sensing platforms for multi-analyte detection/analysis system.

\section{Biochemical activity of the electrowetted TMV-1cys}

Biochemical activity of TMV-1cys assembled by electrowetting on HAR- $\mu$ PAs is evaluated using a sulfhydryl (-SH on cysteine) specific fluorescent labeling reagent, Fluorescein-5Maleimide. Three different conditions are investigated -1) HAR$\mu \mathrm{PAs}$ with electrowetting-assisted assembly of TMV-1cys, 2) HAR$\mu \mathrm{PAs}$ without electrowetting, and 3) planar Au substrate selfassembled with TMV-1cys - to evaluate the wetting process and increased nanoreceptor density by the microstructures.
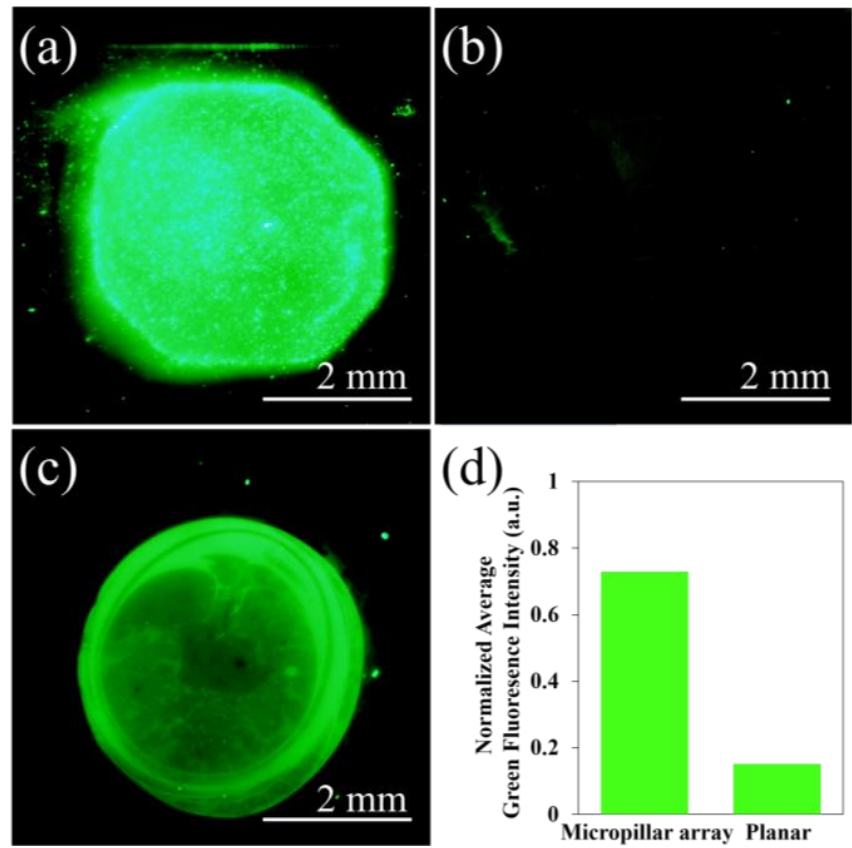

Figure 4. Fluorescence microscopy from (a) electrowetted TMVs on $\mu P A s$, (b) non-electrowetted TMVs on $\mu P A s$, and (c) nonelectrowetted TMVs on planar substrate. (d) Comparison of normalized average green fluorescence intensity between (a) and (c).

Fig. 4a-c compares the fluorescence intensities between the three samples. Fig. $4 \mathrm{a}$ and $4 \mathrm{~b}$ shows the green fluorescence collected from the HAR- $\mu$ PAs with and without electrowetting, respectively. The electrowetted electrodes results in localized TMV functionalization within the wetting boundary while the unwetted electrode barely emits any fluorescence even from the top of the pillars which had been in contact with the TMV droplet during the overnight incubation. This result confirms that the electrowetted TMVs are still chemically functional/selective to maleimides, and the reactivity only remains within the functionalization boundary. The fluorescence intensity from a planar Au electrode is shown in Fig. $4 \mathrm{c}$ where a droplet of TMV solution was placed on the surface overnight for the surface functionalization. Comparing the results with Fig. 4a, the fluorescence intensity is much lower for the planar electrodes with less uniform functionalization of the nanoreceptors over the wetted area. The higher intensity at the wetting edge on planar substrate is attributed to local concentration difference within the droplet due to the surface tension and some liquid drying during the overnight process. Compared to the circular ring on the planar 
substrates, we note that the edge of the functionalization area of the HAR- $\mu$ PAs exhibits angled feature. This is due to the reduced surface continuity for the micropillar arrays compared to the flat surface.

Fig. 4d quantitatively compares the average fluorescence intensities measured from the planar and HAR- $\mu$ PAs electrodes. The intensities collected from the wetted areas are averaged, and normalized by the saturation intensity value (256 bit quantization using ImageJ). A 5-fold increase in fluorescence intensity is achieved for the same exposure time of 2 seconds, which is attributed to an increase in nanoreceptor density within the functionalization area. The enhancement factor from the HAR$\mu$ PAs is slightly less than the theoretically expected value of 6.3. Potential reasons for the results are 1) wider focal depth for the 3D structure compared to the planar substrate, and 2) possible emission losses within the volume of the $\mu$ PAs. Despite the potential limiting factors the technology is still powerful to be implemented for optical sensing applications with the significant increase in the fluorescence intensity.

\section{Localized TMV-1cys functionalization on a single HAR- $\mu$ PAs}

Two droplets of TMV solution with $10 \mu \mathrm{l}$ volume are placed on top of a single $\mu \mathrm{PA}$ electrode $\left(1 \mathrm{x} 1 \mathrm{~cm}^{2}\right)$, and simultaneously electrowetted to demonstrate the advantages of the localized functionalization technique. Only two droplets were able to be located on the same chip due to the limited device footprint and manual control for droplet positioning. A precise control of droplet sizing/positioning on the hydrophobic dense microstructure surface is necessary for reliable implementation of the technology for potential applications.

Fig. 5 shows a fluorescent microscopy taken from the interface of the two functionalization area on a $\mu \mathrm{PA}$ electrode. The very discrete nature of the technique is demonstrated with clear separation between the local fluorescent intensities. The overlaid grayscale analysis further confirms the discrete localization with uniform fluorescence intensity levels confined by sharp edge gradients for both functionalized regions.

The result strongly supports the idea of creating bionanoreceptor functionalized arrayed platform on a single $\mu \mathrm{PA}$ electrode. As discussed above, methods for precise control of droplet positions and sizes along with the advances in chemical/genetic modification technology of bionanoreceptors will deploy this technology for the development of TMV-based multiplexed sensing platform.

\section{CONCLUSIONS}

This work reports a breakthrough in TMV functionalization on HAR- $\mu$ PAs by utilizing electrowetting principles. The electrowetting converts the original limiting principle - structural hydrophobicity of HAR- $\mu$ PAs - to an enabling technology which allows near-complete coating of bioreceptors on high surface area microstructures with localization capabilities.

The 5-fold increase in fluorescence intensity from the hierarchical architecture confirms the biochemical reactivity of the electrowetted TMV-1cys. The demonstrated results are critical evidence that the technology can contribute for the fabrication of high performance (energy/power densities, sensitivities, etc.) microdevices. This opens up new possibilities in micro/nano/bio integrated fabrication technologies and platform for developing miniaturized-systems including micro energy storage devices and biological/chemical sensors.

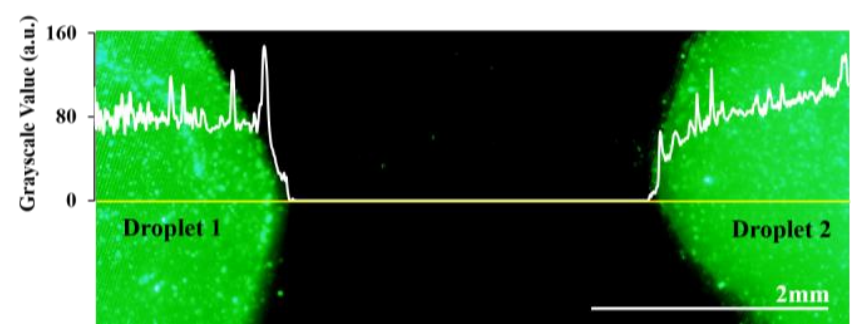

Figure 5. Fluorescence microscopy of two localized TMV functionalizations on a single $\mu P A$ substrate. A line scan measuring grayscale intensity is overlaid.

\section{REFERENCES}

[1] X.Z. Fan, E. Pomerantseva, M. Gnerlich, A.D. Brown, K. Gerasopoulos, M. McCarthy, J.N. Culver, and R. Ghodssi, "Tobacco Mosaic Virus: A Biological Building Block for Micro/Nano/Bio Systems", Journal of Vacuum Science and Technology A, 31, 050815 (2013).

[2] F. Zang, K. Gerasopoulos, X.Z. Fan, A.D. Brown, J.N. Culver, and R. Ghodssi, "Real-Time Monitoring of Macromolecular Biosensing Probe Self-Assembly and On-Chip ELISA Using Impedimetric Microsensors", Biosensors and Bioelectronics, 81, 401 (2016).

[3] X.Z. Fan, L. Naves, N.P. Siwak, A.D. Brown, J.N. Culver, and R. Ghodssi, "Integration of Genetically Modified Virus-LikeParticles with an Optical Resonator for Selective BioDetection", Nanotechnology, 26, 205501 (2015).

[4] F. Zang, K. Gerasopoulos, X.Z. Fan, A.D. Brown, J.N. Culver, and R. Ghodssi, "An Electrochemical Sensor for Selective TMV Sensing Based on Tobacco Mosaic Virus-Like Particle Binding Agents", Chemical Communications, 50, 12977 (2014).

[5] C. Koch, K. Wabbel, F.J. Eber, P. Krolla-Sidenstein, C. Azucena, H. Gliemann, S. Eiben, F. Geiger, and C. Wege, "Modified TMV Partcles as Beneficial Scaffolds to Present Sensor Enzymes", Frontiers in Plant Science, 6, 1137 (2015).

[6] E. Royston, A. Ghosh, P. Kofinas, M. T. Harris, and J. N. Culver "Self-Assembly of Virus-Structured High Surface Area Nanomaterials and Their Application as Battery Electrodes", Langmuir, 24, 906 (2008).

[7] K. Gerasopoulos, E. Pomerantseva, M. McCarthy, A.D. Brown, C. Wang, J.N. Culver, and R. Ghodssi, "Hierarchical Three-Dimensional Microbattery Electrodes Combining Bottom-Up Self-Assembly and Top-Down Micromachining" ACS Nano, 6, 6422 (2012).

[8] S. Chu, K. Gerasopoulos, and R. Ghodssi, "Bio-Nanotextured High Aspect Ratio Micropillar Arrays for High Surface Area Energy Storage Devices", Journal of Physics: Conference Series, 660, 012046 (2015).

[9] A. Tuteja, W. Choi, M. MA, J.M. Mubry, S.A. Mazzella, G.C. Rutledge, G.H. McKinley, and R.E. Cohen, "Designing Superoleophobic Surfaces”, Science, 318, 1618 (2007).

[10] V. Bahadur and S.V. Garimella, "Electrowetting-Based Control of Static Droplet States on Rough Surfaces", Langmuir, 23, 4918 (2007).

\section{CONTACT}

*R. Ghodssi, tel: +1-301-405-8158; ghodssi@umd.com 